Musées, Patrimoine et Culture scientifiques et techniques

$139 \mid 2012$

janvier - février 2012

\title{
Extension du domaine de la taxidermie
}

Extension of the taxidermy field

Jack Thiney et Sophie Grisolia

URL : http://journals.openedition.org/ocim/1012

DOI : 10.4000/ocim.1012

ISSN : 2108-646X

Éditeur

OCIM

Édition imprimée

Date de publication : 1 janvier 2012

Pagination : 5-13

ISSN : 0994-1908

Référence électronique

Jack Thiney et Sophie Grisolia, « Extension du domaine de la taxidermie », La Lettre de I'OCIM [En ligne], 139 | 2012, mis en ligne le 01 janvier 2014, consulté le 02 mai 2019. URL : http://

journals.openedition.org/ocim/1012 ; DOI : 10.4000/ocim.1012 


\section{Extension du domaine de la taxidermie}

\section{Jack Thiney et Sophie Grisolia *}

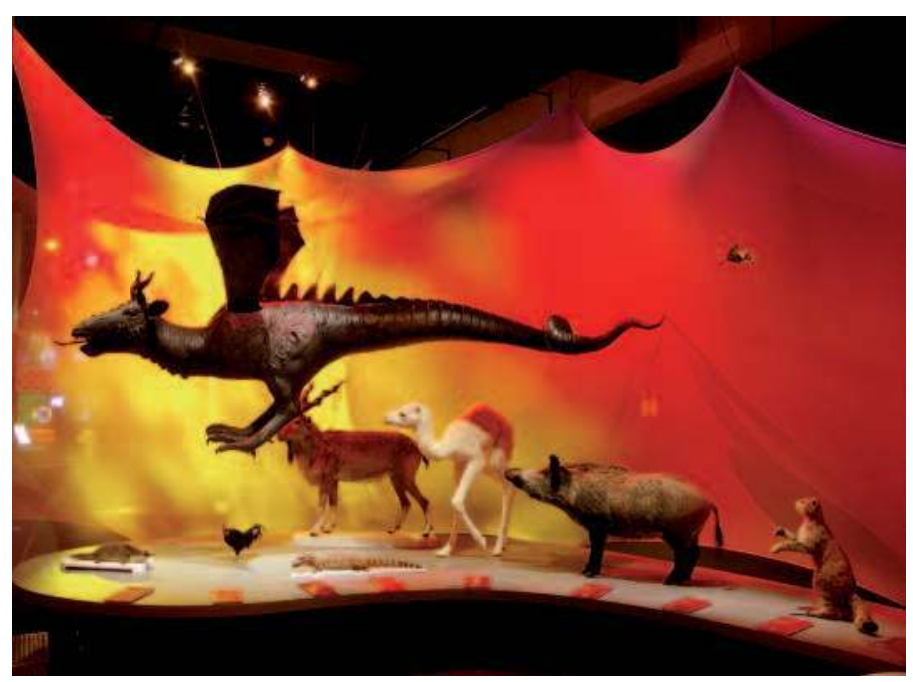

Le dragon imaginé pour l'exposition Dragons, entre science et fiction. () $M N H N / B$. Faye

* Jack Thiney est taxidermiste, ingénieur d'études et spécialiste des grands spécimens au Muséum national d'Histoire naturelle de Paris jackt2@free.fr Sophie Grisolia est muséologue au service des expositions du Muséum national d'Histoire naturelle grisolia@mnhn.fr
Face à la taxidermie traditionnelle, de nouvelles techniques de reconstitution d'animaux disparus et de création de sculptures d'animaux sont apparues ces dernières années : à l'aide d'exemples pris au Muséum national d'Histoire naturelle, les auteurs s'interrogent sur les raisons de cette évolution - liée notamment au développement de la muséologie - et sur le statut (éléments de décors, outils pédagogiques...) de ces nouveaux objets.

Au Muséum national d'Histoire naturelle de Paris, l'édification de nouveaux espaces d'exposition a généré la mise en ouvre de nouvelles collections, qui à leur tour ont favorisé l'émergence de techniques nouvelles.

Cela s'est vérifié lors de l'ouverture de la Galerie de Zoologie de Jules André en 1888. Outre une production frénétique de spécimens, cette époque a permis à une nouvelle technique de voir le jour : l'utilisation du plâtre de Paris pour rendre les volumes. Cette technique, nouvelle pour l'époque, a été adoptée pour la première fois par Jules Terrier, taxidermiste au Muséum, à l'occasion du montage de son buffle d'Afrique (Syncerus caffer caffer) en 1894, toujours visible dans la caravane de la Grande Galerie de l'Évolution. Un peu plus tard, André Boudarel utilisera du grillage et des bandes plâtrées (1948) pour la confection de ses mannequins. Nous lui devons le rhinocéros blanc (Ceratotherium simum), présenté lui aussi à la Grande Galerie. 
Dans le même esprit, la réouverture de la Grande Galerie de l'Évolution en 1994 fut l'occasion, une nouvelle fois, de la création de nombreux spécimens et de l'utilisation de nouvelles techniques. Désormais, les peaux sont posées sur des mannequins en polystyrène et mousse de polyuréthane stratifiés. Depuis sa réouverture, nombre d'anciens spécimens ont été remplacés dans l'exposition permanente par des créations récentes; c'est le cas par exemple du zèbre de Grévy (Equus grevyi), du dromadaire (Camelus dromedarius), du guépard (Acinonyx jubatus), de la hyène (Hyaena hyaena)... et de nouveaux spécimens ont trouvé leur place comme Siam (Elephas maximus) - éléphant d'Asie du zoo de Vincennes -, un tigre de Sumatra (Panthera tigris sumatrae), un anoa (Bubalus depressicornis)... Mais ce qui va surtout changer la demande des concepteurs d'exposition, c'est la création, lors de la rénovation de la Grande Galerie, d'une salle d'exposition temporaire. Ainsi, tous les ans, une nouvelle exposition de $1000 \mathrm{~m}^{2}$ sera produite et le plus souvent déclinée en exposition itinérante à l'issue de sa présentation parisienne.

Chaque exposition implique presque systématiquement de nouvelles créations de spécimens. Peu à peu, la demande des concepteurs va dépasser le cadre de la taxidermie classique en s'étendant vers la reconstitution d'animaux disparus, comme pour l'exposition $A u$ temps des mammouths, jusquà la création de sculptures d'animaux existants comme pour Incroyables cétacés! ou même imaginaires comme pour l'exposition Dragons, entre science et fiction. De plus, un nouvel espace muséal, la Galerie des Enfants qui a vu le jour en juin 2010 , va à son tour être à l'origine d'une demande spécifique : la sculpture d'un éléphanteau d'Asie.

Nous allons montrer, au travers de ces quelques exemples, comment la mise en œuvre de ces différents objets relève à la fois du même protocole de fabrication que celui appliqué à la taxidermie des grands mammifères, mais aussi comment elle en diffère. Nous verrons comment l'évolution dans les musées et la réflexion sur les publics ont généré ces nouvelles demandes. Nous pourrons également nous poser la question du statut de ces nouveaux objets et de leur finalité.

\section{Du grand spécimen à l'artefact : le travail du taxidermiste}

\section{La maquette}

Quel que soit le spécimen ou la sculpture, tout commence par une maquette. La conception d'une maquette à échelle réduite, qui préfigure le mannequin

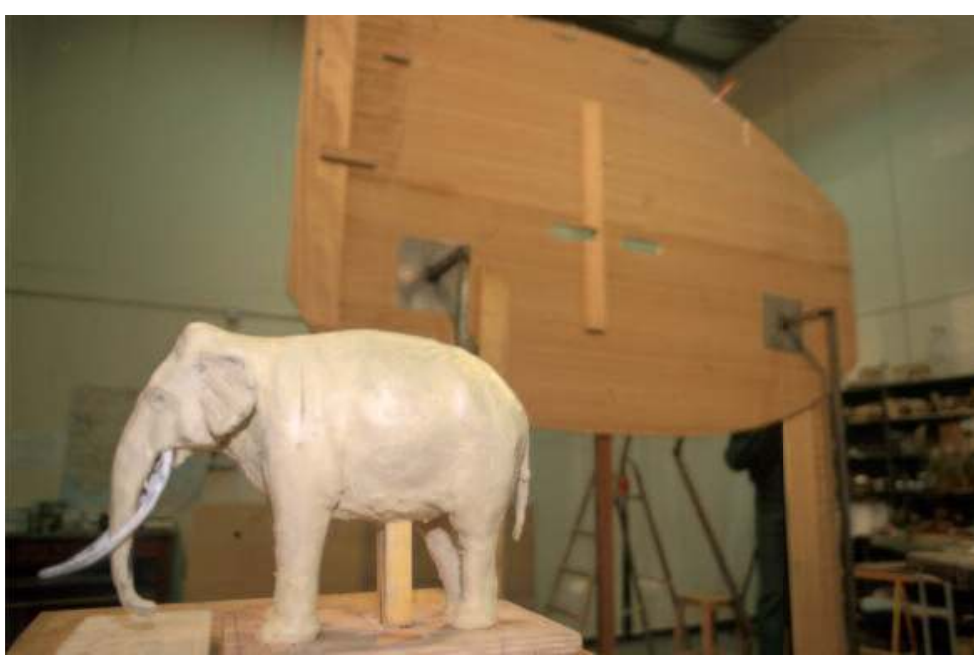

Maquette en plastiline de l'éléphant d'Asie Siam devant la silhouette en bois $\odot M N H N / B$. Faye

définitif, est un pré-requis indispensable à la réalisation d'un spécimen naturalisé, d'une reconstitution (mammouth) ou d'un artefact animalier (tête de cétacé) ou d'une sculpture (dragon).

Il convient tout d'abord de choisir une échelle. Celle-ci est choisie en fonction de la taille du spécimen à représenter : si $1 / 5^{\mathrm{e}}$ convient pour une antilope, $1 / 15^{\mathrm{e}}$ est préférable pour un éléphant. À partir des mesures prises sur l'écorché (l'animal dépecé), la silhouette représentant le corps et la tête de l'animal est découpée dans du bois latté-collé. Les pattes, le cou et la queue sont matérialisés par du fil de fer, qui fera office d'armature. Une potence installée sur le plateau servira à régler la hauteur de l'ensemble. La plastiline, sorte de pâte à modeler qui ne sèche pas, sera choisie pour créer les volumes.

Cette maquette est l'outil indispensable pour le taxidermiste dans son dialogue avec les spécialistes et les muséographes.

\section{Un outil pour le taxidermiste}

Le taxidermiste, dans le meilleur des cas, ne dispose que des mesures prises sur l'animal mort, et après le dépouillage, sur l'écorché. Si précises qu'elles soient, ces mesures sont celles d'un cadavre. Il est donc impératif de les interpréter. À taille réduite, on va donc pouvoir essayer plusieurs options - changer la hauteur du garrot ou allonger le cou, régler les grands volumes, modifier la position... - et ceci avec une grande économie de moyens.

\section{Un outil pour le spécialiste}

Le taxidermiste d'aujourd'hui ne travaille plus seul. La maquette, réalisée en amont de la fabrication du mannequin définitif, va être soumise aux spécialistes 
de l'animal, quiils soient soigneurs, vétérinaires ou chercheurs, car leur expertise est précieuse. Le taxidermiste sera donc en mesure de prendre en compte leurs observations et d'apporter les modifications nécessaires sur la maquette.

\section{Un outil pour le muséographe}

La maquette est réalisée à une échelle donnée. Il suffira de multiplier les dimensions de celle-ci par le coefficient d'agrandissement pour appréhender l'encombrement de l'objet à l'échelle $1 / 1$. À son tour, le muséographe aura la possibilité d'exprimer ses choix. Il pourra formuler ses exigences en matière de posture et de positionnement du spécimen dans l'exposition ; le scénographe, lui, pourra organiser le mobilier. Grâce à la prise de vue numérique, ce dernier sera en mesure d'intégrer le spécimen dans son logiciel de modélisation en 3D et obtenir ainsi une préfiguration de l'objet dans l'exposition.

\section{Siam, éléphant d'Asie : la taxidermie classique d'un grand spécimen}

La peau de ce spécimen hors du commun a été prélevée le jour même de son euthanasie (24 septembre 1997). Cet animal, d'un poids de 6 tonnes, a nécessité pas moins de dix personnes pour procéder à son dépouillage.

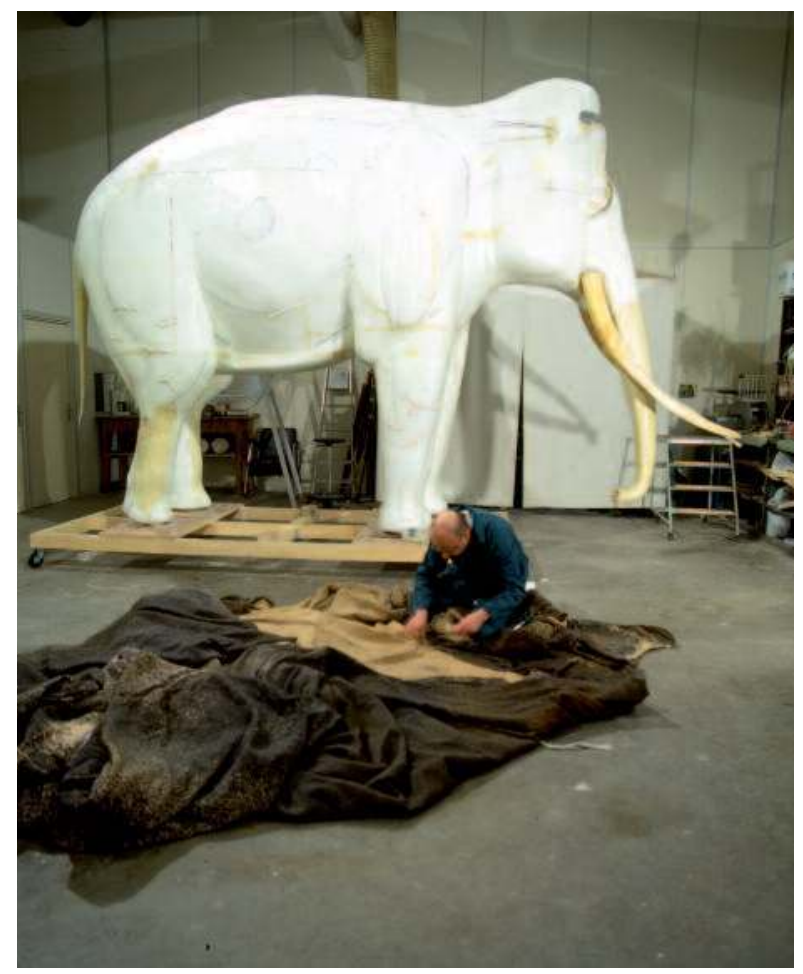

Couture sur la peau tannée de Siam devant le mannequin en polystyrène (c) $M N H N / B$. Faye
Sa peau a été confiée aux bons soins d'un tanneur. Au terme de quelques mois, il l'a rendue tannée, c'està-dire imputrescible, souple, et surtout beaucoup plus légère puisqu'elle ne pesait plus que $160 \mathrm{~kg}$ au lieu des $600 \mathrm{~kg}$ initiaux.

Une maquette en plastiline au $1 / 15^{\mathrm{e}}$ a été réalisée à partir des croquis et mesures pris sur l'animal, son écorché et aussi son squelette. Après qu'elle ait été validée par les scientifiques, ainsi que par les responsables de la muséographie ayant en charge l'exposition permanente, il a été procédé à la fabrication du mannequin selon la technique du mannequin rigide. Il s'agissait en fait de pratiquer un agrandissement de la maquette pour parvenir à l'échelle $1 / 1$. Les silhouettes de la tête et du corps sont découpées dans du bois latté-collé de $20 \mathrm{~mm}$ d'épaisseur. Les armatures des pattes ainsi que les platines soutenant les défenses sont en métal (tube carré). Elles sont soudées grâce à un appareil de soudure à l'argon qui diminue de façon sensible le dégagement de chaleur. Il permet ainsi de réaliser des soudures à proximité de matériaux inflammables (bois, polystyrène...) et évite les déformations du métal. Des plaques de polystyrène (densité $25 \mathrm{~kg}$ au $\mathrm{m}^{3}$ ) ou de la mousse de polyuréthane sont ensuite posées sur la silhouette pour créer les volumes. Ces matériaux sont alors sculptés afin d'obtenir le rendu des masses musculaires.

Le mannequin est ensuite stratifié au moyen de résine époxy et de tissu de verre. Cette technique permet d'obtenir une coque fine et résistante qui augmentera la solidité de l'ensemble et, par là même, la pérennité du spécimen. Enfin, la peau est collée sur le mannequin et les coutures des incisions pratiquées pendant le dépouillage sont cousues. Des yeux de verre sont mis en place et contribueront à donner líllusion de la vie. Après séchage, une légère mise en couleur est pratiquée au moyen d'un aérographe de façon à rehausser les pigments altérés lors du tannage.

La naturalisation de Siam est un exemple type de taxidermie. C'est cette méthode qui sera déclinée pour la conception et la réalisation des nouveaux artefacts demandés par les concepteurs d'exposition.

\section{Mammouth : la reconstitution d'un animal disparu}

C'est dans le cadre de l'exposition temporaire Au temps des mammouths, qui a eu lieu de mars 2004 à janvier 2005 à la Grande Galerie de l'Évolution, qu’a été réalisée la reconstitution échelle 1 de cet animal disparu. 


\section{Spécificité de la demande}

Il s'agissait de reconstituer un mammouth mâle adulte (Mammuthus primigenius), taille réelle $(3,40 \mathrm{~m}$ au garrot), correspondant aux critères scientifiques actuels : justesse des proportions et de l'attitude, pose d'une toison la plus réaliste possible. En prévision de l'itinérance de l'exposition, le cahier des charges spécifie également que le spécimen doit être entièrement démontable, afin de permettre son passage par une porte standard.

\section{Réalisation}

Le protocole de fabrication utilisé pour la naturalisation de Siam est décliné pour la reconstitution de

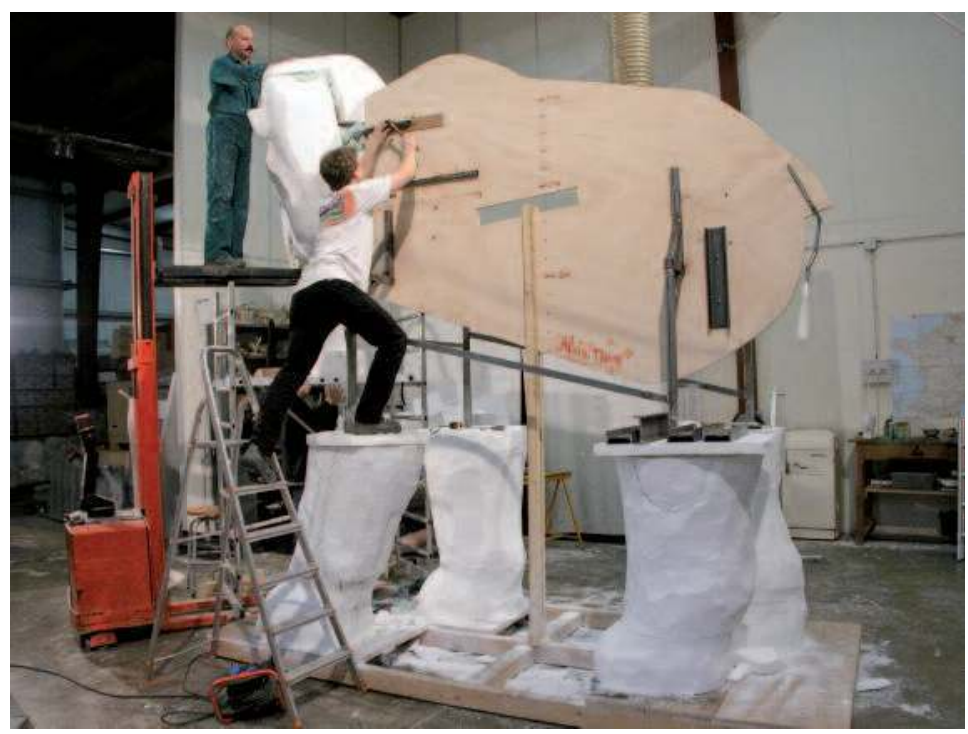

Positionnement de la tête du mammouth sur la silhouette en bois par Jack Thiney et Antoine Jayez (C) MNHN/B. Faye

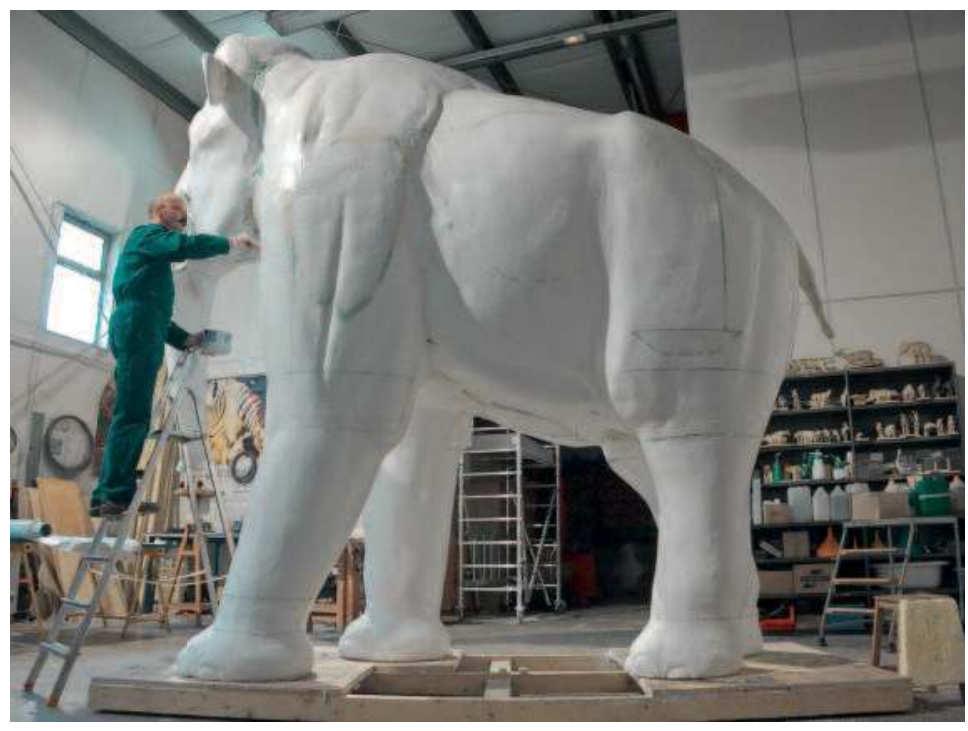

Stratification du mannequin (๑) $M N H N / B$. Faye ce mammouth. La différence majeure réside dans le fait que l'on ne dispose pas, comme pour l'éléphant, du cadavre de l'animal et que l'on est donc dans l'impossibilité de prendre les mesures sur l'écorché. Dans ce cas, le recours à l'étude d'un squelette monté est sans conteste la meilleure solution. Celui du musée d'Histoire naturelle de Bruxelles correspondait à la taille recherchée. Il a fourni une partie des informations manquantes recueillies par le biais de croquis détaillés, de mesures et de photographies. Par ailleurs, les paléontologues spécialistes du mammouth ont fourni un dessin scientifique du squelette en situation de marche.

C'est grâce à l'ensemble de ce matériel que la maquette à l'échelle $1 / 16^{\mathrm{e}}$ est réalisée. Elle est proposée aux chercheurs et aux muséographes pour être validée. Les modifications éventuelles sont apportées et ce n'est qu'avec l'accord de toutes les parties que la construction peut commencer.

La conception du mannequin est identique à celle de Siam, outre le fait que ce mannequin doit être démontable, ce qui implique d'en tenir compte dès la mise en œuvre de la structure métallique. Les pattes, la trompe, les défenses ainsi que le flanc gauche ont donc été conçus pour être amovibles.

Il ne reste plus alors qu'à poser la fourrure synthétique. La surface stratifiée autorise l'utilisation d'une agrafeuse électrique. Deux qualités de fourrure sont installées : l'une, à grande longueur et à haute densité de poils, est posée sur le dos, le dessus de la tête et les flancs de l'animal ; l'autre, à faible longueur et à basse densité de poils, est réservée pour le dessous du corps et pour la trompe.

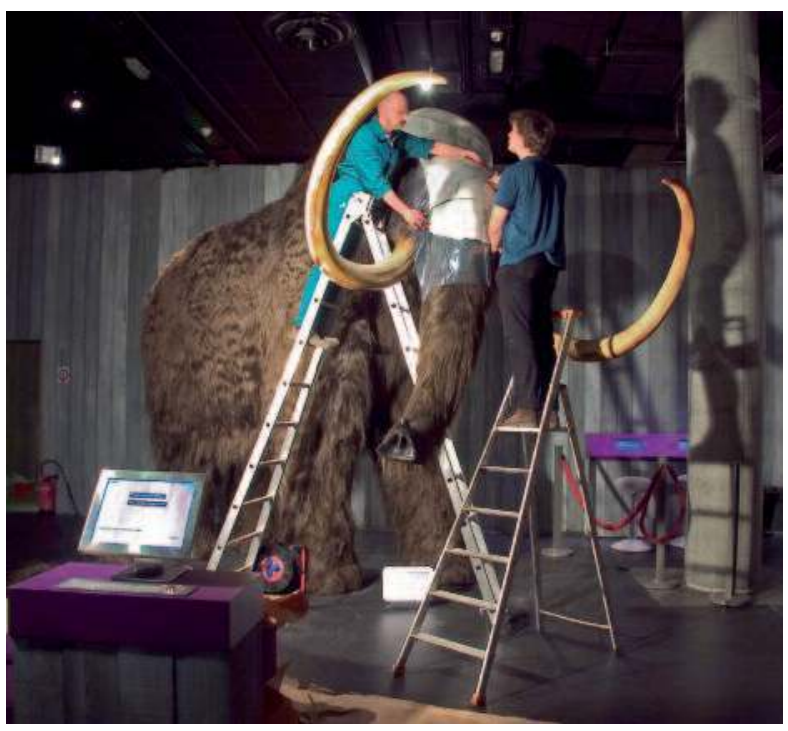

Pose de la fourrure synthétique (๑) $M N H N / B$. Faye 
Date : 8 avril 2004

\section{Fiche Mammouth Mammuthus primigenius}

Travaux à mettre en ouvre : reconstitution à partir d'une maquette au 1/16e d'un mammouth mâle taille adulte (3,40 m), qui puisse être démonté de façon à passer par une porte de 2,50 m x 1,45 m. Parties démontables : les pattes, les défenses, la trompe, le côté gauche (accroche par trois platines en contreplaqué), le fourreau. Quatre platines métalliques permettront son installation sans la nécessité d’un socle.

Intervenants : Jack Thiney assisté de Antoine Jayez. Pose de la fourrure synthétique (40 h). Suivi scientifique effectué par MM. Tassy, Lester et Schomani.

Dimensions : H 3,60 m, L 1,60 m

Numéro de transpondeur : 00001299 D04

Produits employés : contreplaqué : 4,50 $\mathrm{m}^{2}$, tube carré de $35 \mathrm{~mm}$, et $40 \mathrm{~mm}$, tube rond de $30 \mathrm{~mm}$. Tôles 20 x $10 \mathrm{~cm}$. Polystyrène M3 $20 \times 3 \mathrm{~cm} \times 1,8 \mathrm{~m}^{3}$. Mousse de polyuréthane en bombe (45). Colle acrylique. Défenses : 2,80 m, en résine époxy modelable axson sc 258. Finition par glacis au vernis acrylique et aérographe. Encre sépia colorex 34T.

Temps de travail : mannequin 4 mois

Coût fourniture mannequin : $3000 €$

Fourrure synthétique : 363 sq foot de 10 à 12 pouces long hair extra dence + under ur 132 sq foot same color.

Coût : $20000 €$

Fournisseur : Rémie Bakker Librijesteeg 1473011 HN Rotterdam Mél : info@manimalworksorks.com tél. : + 31104129938 cell. + 31627476053

Destination : exposition Au temps des mammouths

Exemple de fiche de réalisation

\section{Dragon : la création d'une créature légendaire}

C'est dans le cadre de l'exposition Dragons, entre science et fiction, qui s'est déroulée à la Grande Galerie de l'Évolution du 5 avril au 6 novembre 2006, qu'a été réalisée cette chimère de grande taille.

\section{Spécificités de la demande}

Il s'agissait de réaliser un dragon de $5 \mathrm{~m}$ qui devait être démontable. Il était composé des éléments d'une douzaine d'animaux réels : tête de dromadaire, ailes de chauve-souris, oreilles de cochon, crête d'iguane, langue de serpent, pattes de coq, peau de crocodile, dents de tigre, cornes de markhor, moustaches de chat et griffes d'aigle.

\section{Réalisation}

Pour composer la chimère, le recours à l'iconographie demeure la seule alternative. Un croquis est esquissé dans lequel figurent les animaux sélectionnés. À partir de cette ébauche, trois maquettes différentes sont proposées aux muséographes. L'une d'entre elles a été validée au terme d'âpres négociations!
La construction du mannequin suit le même protocole que les exemples précédents. Des platines métalliques sont prévues pour le démontage des ailes et de la queue. Sur cette structure complexe, on utilise la mousse de polyuréthane pour créer les volumes plutôt que les dalles de polystyrène.

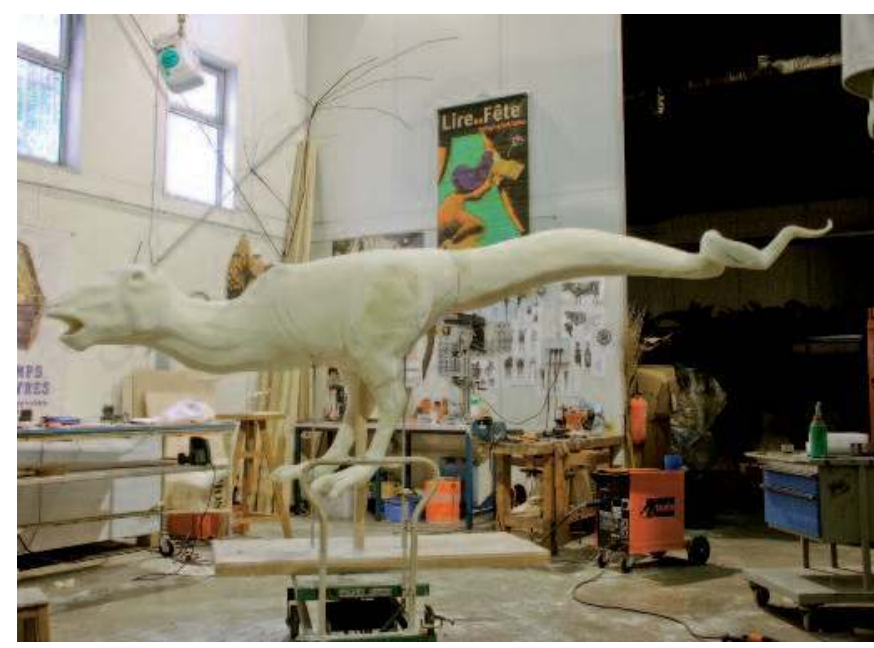

Mannequin du dragon en mousse de polyuréthane ○ MNHN/B. Faye 
Le mannequin est ensuite stratifié, le rendu de la texture de la peau peut alors être mis en œuvre. Il faudra de nombreuses investigations en zoothèque, au salon du cuir et dans les pelleteries pour trouver le motif adéquat. Finalement, c'est le dos d'un caïman noir provenant des collections du Muséum qui fournit les différents motifs servant à recouvrir le corps du dragon. Les tirages en résine époxy sont démoulés avant la fin de la polymérisation afin que chaque moulage épouse son nouveau support.

Le relief de la peau des pattes est obtenu par modelage. Les ailes sont construites sur le modèle de celles des chauves-souris. À partir d'une structure en métal soudé, la voilure est découpée dans du carton, mise en forme, stratifiée, puis enduite et poncée. On procède de la même façon pour réaliser la crête dorsale et les oreilles du dragon. Des dents de tigre ainsi que des cornes de markhor sont moulées puis tirées en résine polyuréthane. Toutes les différentes pièces sont ensuite raccordées entre elles par modelage. Enfin, la mise en teinte est réalisée au moyen de bombes aérosol pour la couleur de fond et à l'aérographe pour les finitions.

\section{Cétacés : sculptures hyperréalistes d'animaux impossibles à naturaliser}

C'est dans le cadre de l'exposition temporaire Incroyables cétacés! organisée au Muséum de Paris du 11 juin 2008 au 25 mai 2009 qu'ont été réalisées ces sculptures de mammifères marins.

\section{Spécificités de la demande}

Trois têtes de cétacés sculptées à taille réelle, comme sortant de l'eau : narval (Monodon monoceros),

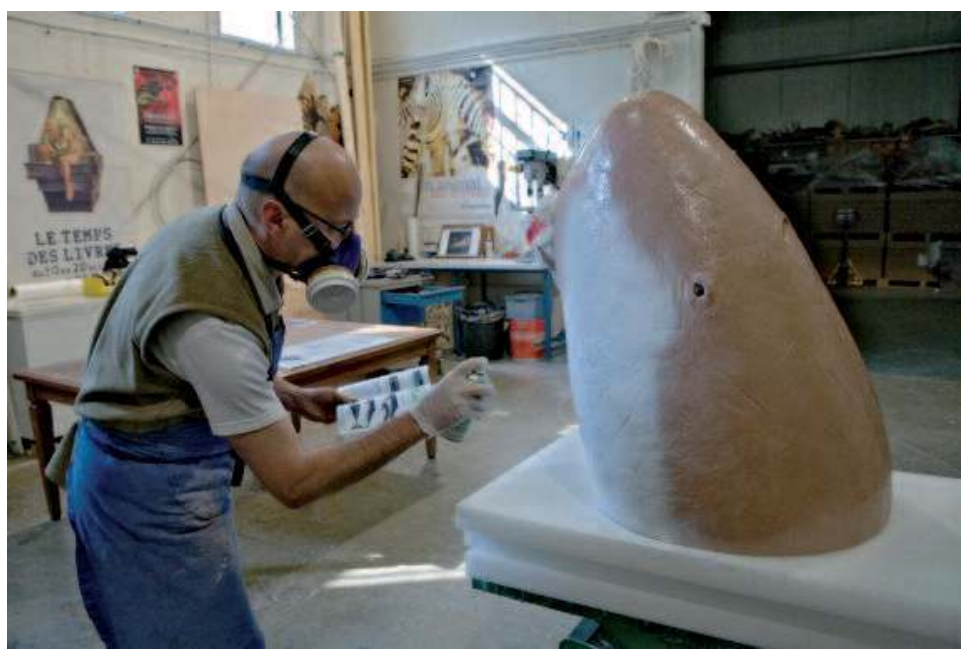

Mise en couleur à l'aérographe du cachalot pygmée destiné à l'exposition Incroyables cétacés! (C) MNHN/B. Faye baleine à bec (Mésoplodon layardii), cachalot pygmée (Kogia breviceps). L'objectif est de réaliser des sculptures hyperréalistes et pouvant être caressées par les visiteurs. La sensation au toucher doit se rapprocher de l'effet «botte mouillée».

\section{Réalisation}

Nous ne disposons évidemment pas de tels spécimens à naturaliser. De plus, le choix de la taxidermie pour la représentation de cétacés est très discutable. En effet, le tannage de ces peaux extrêmement grasses n'est jamais très satisfaisant, ce qui implique inévitablement d'avoir recours à un processus de restauration de la peau par enduit/ponçage et mise en couleur. On aboutit alors à un surfaçage entièrement synthétique, et la peau disparait sous les couches d'enduit et de résine... La fabrication d'un artefact est donc préférable. Le principe de réalisation est similaire à celui des exemples précédents : une maquette est soumise à la validation des muséographes et des scientifiques. Elle précède la mise en œuvre d'un mannequin rigide en mousse de polyuréthane stratifié. Les pièces dentaires - la dent du narval, la mâchoire du cachalot et les dents du mésoplodon - sont moulées et tirées en résine puis patinées.

En ce qui concerne le surfaçage, le narval et le mésoplodon subissent le même traitement. Il s'agit d'obtenir un effet lisse, glissant, évoquant l'hydrodynamisme de ces animaux. La surface est enduite et poncée jusqu'à ce que toute imperfection disparaisse. La mise en couleur est obtenue au moyen de glacis successifs. La dernière touche est donnée par la pose d'une résine transparente et souple au toucher qui contribue à restituer l'effet «botte mouillée » demandé. Concernant le cachalot pygmée, on utilise le report d'empreinte pour restituer le relief plus marqué de sa peau. La résine transparente est ensuite appliquée en dernière couche pour obtenir une homogénéité de rendu avec les autres sculptures de cétacés.

\section{L'éléphanteau : I'objet que l'on peut toucher}

C'est dans le cadre de la création de la Galerie des Enfants, espace permanent à destination des 6-12 ans, situé dans la Grande Galerie de l'Évolution, qu'a été réalisée la sculpture d'un éléphanteau d'Asie (Elephas maximus)

\section{Spécificité de la demande}

Produire une sculpture hyperréaliste destinée à être touchée par les enfants. Ceci implique la réalisation d'un moulage en résine teintée dans la masse. Le spécimen sera fixé directement sur le sol (absence de socle). 


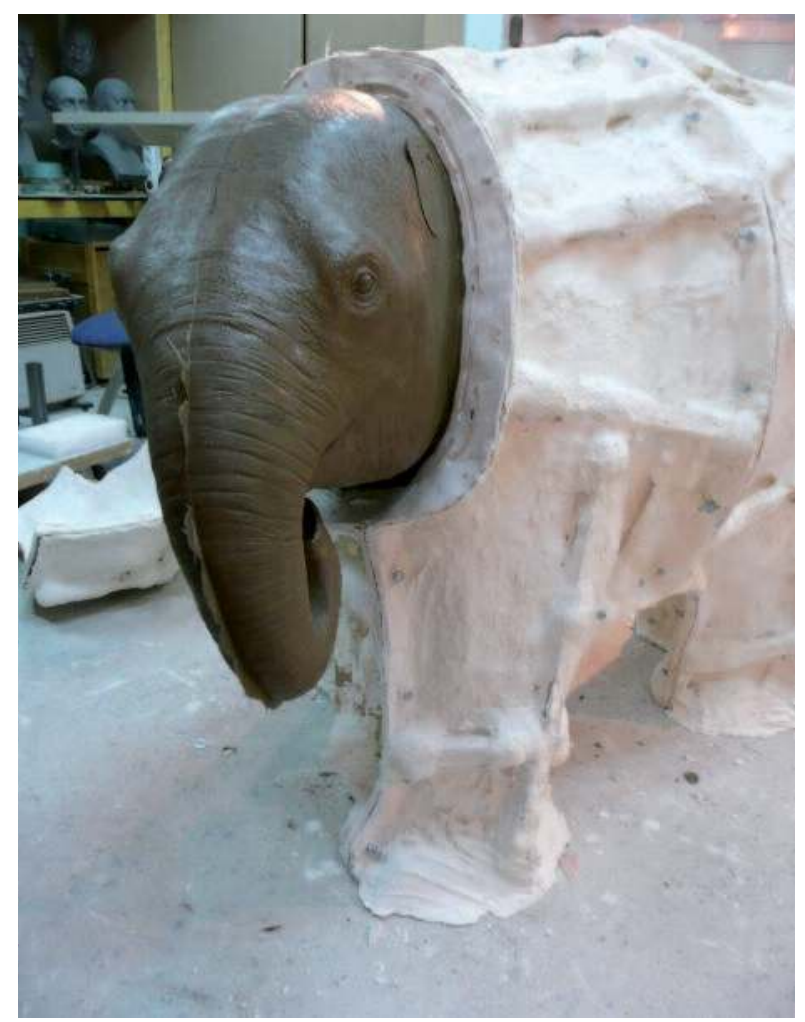

L'éléphanteau dans l'atelier du mouleur Stéphane Barret ○) S. Barret

\section{Réalisation}

Après avoir exploité les ressources de l'iconographie (photographies, vidéo...), plusieurs maquettes proposant des postures différentes ont été créées et soumises aux muséologues en charge du projet. L'une d'entre elles a été retenue et validée.

Un mannequin rigide en polystyrène et mousse de polyuréthane stratifié est construit sur le même modèle que celui des exemples précédents.

Après stratification, la peau est créée au moyen d'une fine couche de pâte époxy. Le relief de la peau est alors recréé au moyen de tampons appliqués sur cette pâte très modelable. Une fois terminée, cette sculpture sert de matrice au moulage et est soumise à la validation des responsables de l'exposition. Elle est alors confiée aux bons soins d'un mouleur qui produit un tirage en résine chargée en bronze. De cette manière, l'objet touché se patine au fil du temps, les zones les plus touchées devenant plus brillantes, comme on peut l'observer sur un vrai bronze. Des platines métalliques intégrées sous les pattes permettent sa fixation directement sur le sol.

Après cet aperçu des différentes techniques utilisées pour réaliser ces nouveaux objets muséaux, on peut se poser la question de l'origine de ces demandes, et de la place et de l'avenir de ces artefacts.

\section{Pourquoi ces nouvelles demandes ?}

Depuis quelques dizaines d'années, les expositions se sont multipliées et le monde des musées s'est professionnalisé. Par ailleurs, avec le développement de la muséologie, le musée réfléchit, se pose de plus en plus de questions sur lui-même, sur son évolution, sur la place et l'usage de ses collections, mais aussi sur ses messages, sur ses visiteurs et sur ses objectifs. Quels sont nos publics? Que voulons-nous leur dire, leur montrer ? Que viennent-ils chercher au musée ?

C'est dans ce contexte d'évolution de la muséologie et de la muséographie que se multiplient ces artefacts muséaux.

\section{Évolutions des objets au sein de l'exposition scientifique}

Désormais, le spécimen ou l'objet de collection n'est plus le seul support de sens au musée. Avec le passage du «musée d'objets » au «musée d'idées », le spécimen n'est plus seulement là en tant qu'objet de collection et représentant d'une espèce, il devient aussi le support d'un message, le prétexte à un discours.

Dans cette nouvelle tendance où le discours guide la conception muséologique, que faire en l'absence de spécimens? Certes, le développement de la muséographie a intégré les nouveaux médias comme l'iconographie, l'audiovisuel ou le multimédia. Mais peu à peu, en l'absence d'objets de collection, l'artefact, le moulage ou la reconstitution sont venus prendre une place de plus en plus importante dans les expositions temporaires. On s'autorise ainsi une sculpture hyperréaliste pour suppléer l'absence d'un animal naturalisé, comme pour l'exposition Incroyables cétacés!, et l'on ose reconstituer échelle 1 l'animal disparu comme pour l'exposition Au temps des mammouths.

Tout comme le spécimen, ces sculptures offrent l'avantage d'être tridimensionnelles et de donner la véritable échelle au visiteur, ce qu'une photographie ou une vidéo ne donneront jamais. Le médium exposition conserve alors le fondement sur lequel il repose: l'objet... si particulier soit-il.

\section{Évolution des publics \\ et de leur prise en compte}

C'est aussi par l'étude et la prise en compte des différents publics que la muséographie a cherché à adapter les supports du discours, pour le rendre plus accessible et plus attractif. Les expositions scientifiques ont pour public privilégié les familles et les scolaires ; l'enfant est donc un visiteur cible. Peut-on concevoir une exposition sur les mammouths ou sur les cétacés en ne montrant que des squelettes? En l'absence de spécimens, ces 


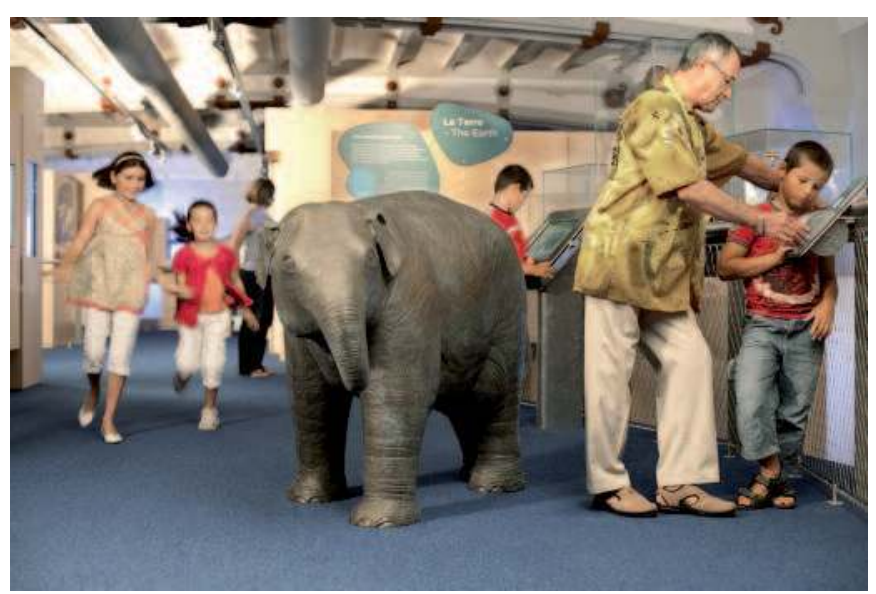

L'éléphanteau dans la Galerie des Enfants du Muséum national d'Histoire naturelle ○ MNHN/M. Voyeux

sculptures ont un rôle didactique qui permet de montrer l'animal dans son intégralité et à sa véritable échelle. Par ailleurs, ces objets sont d'autant plus attractifs que certains sont conçus pour pouvoir être touchés, ce qui est impossible avec un spécimen naturalisé. C'est précisément dans cette optique que fut réalisé l'éléphanteau de la Galerie des Enfants. Aujourd'hui, on ne vient plus seulement au musée pour voir, on peut interagir, écouter, faire, jouer et parfois même... on peut toucher. Cela participe à rendre le musée plus accessible, à le désacraliser pour les plus jeunes, sans pour cela mettre en danger les collections. La place de l'objet de collection reste claire : on ne touche pas les « vrais ».

La volonté de présenter de tels objets et de pouvoir les rendre « touchables » naît aussi de la récente prise en compte des publics handicapés, en particulier les aveugles. Le mammouth ne pouvait être touché car les poils de la fourrure synthétique restaient trop fragiles, cependant linterdiction était levée dans le cadre des visites accompagnées pour les aveugles. En ce qui concerne les têtes de cétacés et l'éléphanteau de la Galerie des Enfants, ils ont dès le départ été conçus pour être accessibles à tous les visiteurs, et pour être des éléments phares du parcours de visite destiné aux non voyants.

\section{Évolutions de la nature des expositions et de leur devenir}

Parallèlement au développement des expositions temporaires, la demande de versions itinérantes a vu le jour. Très rapidement se pose alors la question du déplacement des collections : faire voyager pendant plusieurs années des spécimens datant pour la plupart du XIXe siècle est impossible, encore moins s'il s'agit de grands spécimens. L'utilisation de ces sculptures permet de maintenir la présence d'objets tridimensionnels dans les expositions itinérantes, mais aussi de conserver des points forts de la visite. Ces artefacts étant spécifiquement créés à l'occasion du projet, ils peuvent intégrer tous les impératifs liés à litinérance : légèreté, maniabilité pour le transport, démontabilité, installation rapide avec des supports sur platine, résistance... Ainsi, le mammouth voyage en huit morceaux, les raccords sont masqués par la longueur de ses poils ; la tête de narval a été prévue avec une défense démontable, tout comme la queue du dragon.

On s'aperçoit donc que la commande de ces artefacts naît de la volonté des concepteurs de rendre toujours plus accessible et plus attractive l'exposition auprès de ses différents publics. Ceci, tout en conservant ce qui fait l'essence même de l'exposition, l'objet. On peut alors se poser la question : puisqu'il ne s'agit pas de "vrais » spécimens, pourquoi faire appel à un taxidermiste ?

\section{Pourquoi s'adresser à un taxidermiste?}

C'est en s'intéressant au travail du taxidermiste, et en l'observant lors de la réalisation de nouveaux spécimens, tel Siam, que les concepteurs d'exposition ont pris conscience que les techniques et les étapes du processus étaient en partie communes avec le travail d'un sculpteur animalier : documentation, observation, croquis puis maquette et enfin sculpture échelle 1.

Le taxidermiste, le concepteur et le scientifique partagent des habitudes de travail. Ils ont aussi la même volonté d'être le plus juste scientifiquement dans ce qu'ils délivrent au public, dans la nécessité de se rapprocher le plus possible de la réalité.

Face à cette exigence partagée, le taxidermiste apparaît être pour le concepteur le meilleur artisan de ces artefacts, reconnu par l'institution et les scientifiques. Outre des compétences de sculpteur, on s'assure de méthodes de travail rigoureuses qui s'appuient sur la bibliographie scientifique, la présence et les validations des spécialistes, des collections de référence pour prendre toutes les mesures ou faire les moulages nécessaires.

Dans cette course pour se rapprocher du "vrai », qu'en est-il finalement du statut de ces objets, qu'en pense le musée, qu'en pense le public ?

\section{Quel est le statut de ces nouveaux objets?}

Malgré l'énergie déployée pour se rapprocher de la réalité, ces objets restent objectivement «faux » pour l'ensemble des acteurs. Mais cela veut-il dire pour autant qu'ils n'ont pas de valeur?

Pour le conservateur, ou le chargé de collections, ils n'ont pas de valeur car ils ne sont pas « vrais ». Pour certains, ces objets sont même relégués au rang d'éléments 


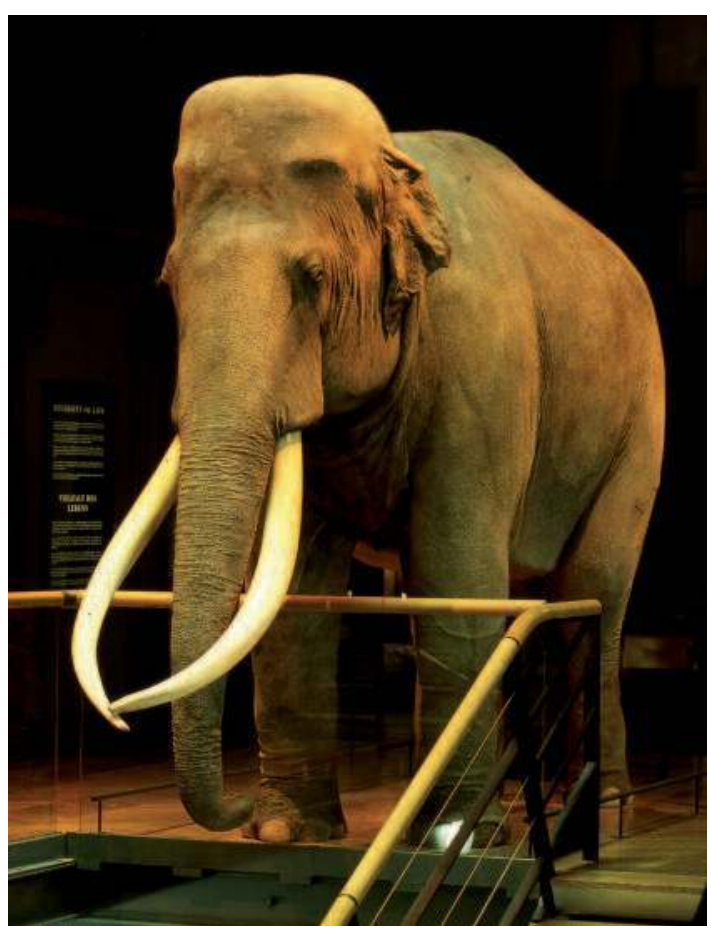

L'éléphant d'Asie Siam dans l'exposition permanente du Muséum national d'Histoire naturelle (c) MNHN/B. Faye

de décors. N'est-il pas plus juste de dire qu'ils n'ont pas de valeur aujourd'hui ? Ainsi, la reconstitution en plâtre du Dodo, réalisée par Jules Terrier en 1905, n’a-t-elle pas acquis une valeur historique?

Pour le concepteur, le regard est différent. Ces objets ont une valeur pédagogique : ils donnent à voir l'animal, et permettent de prendre conscience de sa taille réelle. Ils ont aussi une valeur muséographique : ce sont le plus souvent des «objets phares ", c'est-à-dire qu'ils marquent un moment important dans le parcours de visite. Ils ont une valeur émotionnelle, espère-t-on, en suscitant peut-être l'étonnement ou le questionnement, au moins pour la taille.

Quoi qu'il en soit, ces artefacts ou ces reconstitutions n'ont pas vraiment atteint les expositions permanentes (même le calmar géant a été remplacé par un spécimen plastinisé). On accepte le moulage qui est une fidèle réplique du « vrai », on autorise la maquette de dinosaure, qui accompagne le vrai et grand squelette; mais le mammouth reconstitué ou la tête de baleine n'ont pas leur place dans l'exposition permanente. Seul l'éléphanteau a franchi la porte... mais il est dans la Galerie des Enfants où il y a plus de dispositifs que de spécimens.

Et pour le visiteur, justement, quelle place, quelle valeur ont ces objets ? Ces objets ne posent pas seulement l'éternelle question du vrai ou du faux dans le musée. Pour Karine Langlois, qui a étudié l'artificialisation des objets de sciences naturelles, " on ne peut opposer l'artefact au naturel. Les visiteurs ont même parfois du mal à distinguer le vrai du faux au niveau des collections qui leur sont présentées, ces objets s'intégrant souvent parfaitement au sein du discours d'exposition » (1). Pour les artefacts de grande taille, comme ceux qui nous occupent, il y a peu de doute sur la nature de l'objet pour le visiteur, et dans ce cas, "l'usage de fanx, au sein d'une exposition, est bien accepté. Le choix du concepteur est compris par la plupart des visiteurs et le message semble passer dans la majorité des cas ».

Lorsque l'on regarde les évaluations réalisées sur ces expositions par Frédérique Lafon (responsable de l'Observatoire des publics du MNHN), ces artefacts ne sont pas forcément cités nominativement dans les éléments marquants de l'exposition, (le mammouth est cité avec son dispositif qui permettait de se prendre en photographie avec l'animal) mais, on ne les retrouve pas, non plus, dans les éléments de déception. En revanche dans l'exposition Dans l'ombre des Dinosaures, certains visiteurs auraient apprécié de voir quelques reconstitutions accompagner les squelettes de dinosaures.

Tout ceci laisse donc à penser que les visiteurs interprètent parfaitement ces objets et décodent les intentions pédagogiques qui les accompagnent.

\section{Conclusion}

L'évolution de la muséologie, de concert avec celle de la taxidermie, a conduit le taxidermiste à créer de nouveaux objets qui relèvent plus de la muséographie que des collections. Le musée n'est pas encore prêt à les assumer dans ses réserves ou dans ses expositions permanentes. Aujourd'hui, ces artefacts figurent davantage dans les expositions temporaires ou itinérantes ; mais après, que deviennent-ils ? On ne garde pas les autres supports muséographiques, mais ces objets ont aussi un coût non négligeable. Faut-il les conserver ? Qui se charge de les stocker et où ? À l'heure où le mètre carré vaut de l'or, il devient difficile de leur trouver une place. Quelle valeur auront-ils dans un siècle ou deux ? Pour qui ? Auront-ils plus de valeur si l'espèce qu'ils représentent disparait ? Devra$\mathrm{t}$-on les conserver, voire les restaurer?

\section{Note}

(1) Langlois, K. L'objet de musée entre nature et artifice, entre discours et visiteurs : l'artificialisation des objets de sciences naturelles et ses conséquences sur la médiation muséale. Thèse de Doctorat, sous la direction du professeur Patrick Blandin, 2005. 